\title{
Comprovando o volume da esfera nas aulas de matemática do Ensino Médio
}

\author{
Rudimar Luiz Nós" \\ DAMAT/UTFPR, Curitiba, PR \\ Maria Carla F. P. Tavares $\mathbb{R}^{2}$ \\ CPM - Colégio da Polícia Militar do Paraná, Curitiba, PR
}

\begin{abstract}
Resumo. Apresentamos neste trabalho duas estratégias para provar a relação para o cálculo do volume da esfera no Ensino Médio: o princípio de Cavalieri e a lei da alavanca de Arquimedes. Empregamos o software gratuito GeoGebra 3D para comprovar a relação para o volume dinamicamente e propomos atividades para a sala de aula baseadas nessas duas estratégias. Concluímos que o aplicativo de geometria dinâmica é uma ferramenta eficaz para construir figuras bidimensionais e tridimensionais, bem como para comparar áreas e volumes dessas figuras.
\end{abstract}

Palavras-chave. A lei da alavanca de Arquimedes, O princípio de Cavalieri, GeoGebra 3D, Ensino de Matemática, ENEM.

\section{Introdução}

Nas últimas décadas, as reformas educacionais propostas para o ensino de matemática na Educação Básica evidenciam a importância do ensino de geometria plana e geometria espacial. A Base Nacional Comum Curricular (BNCC) 4 de matemática para o Ensino Fundamental enfatiza o desenvolvimento de competências através de cinco unidades temáticas correlacionadas, sendo a Geometria uma delas. Para o Ensino Médio, a BNCC estabelece como competência específica 5 para Matemática e suas Tecnologias:

Investigar e estabelecer conjecturas a respeito de diferentes conceitos e propriedades matemáticas, empregando estratégias e recursos, como observação de padrões, experimentações e diferentes tecnologias, identificando a necessidade, ou não, de uma demonstração cada vez mais formal na validação das referidas conjecturas (BRASIL, 2018, p. $540)$.

A geometria é um conteúdo presente em todos os documentos que orientam o planejamento e o desenvolvimento da matemática nos vários níveis educacionais [4, 16], sendo aplicada tanto de forma direta quanto transversal, de maneira a contribuir para que o estudante desenvolva uma visão espacial. Além disso, é base de conhecimento para outras áreas da ciência e tecnologia, como por exemplo, a física e as engenharias, reforçando seu caráter multidisciplinar no processo educacional. Tal importância é evidenciada pela quantidade significativa de questões de geometria plana e de geometria espacial presentes no Exame Nacional do Ensino Médio (ENEM). Nos mais de vinte anos de existência desse exame, podemos elencar na prova de Matemática e suas Tecnologias muitas questões envolvendo o cálculo de áreas e de volumes [12 13].

\footnotetext{
${ }^{1}$ rudimarnos@utfpr.edu.br.

${ }^{2}$ maria_carla24@yahoo.com.br.
} 
Nas questões de geometria espacial do ENEM, destacamos várias abordando a esfera [19]. Essas questões são tanto de cunho aplicado, como o cálculo do volume - Figura 1 (a), quanto de cunho conceitual - Figura 1(b). A questão ilustrada na Figura 1(b) aborda um aspecto da demonstração da relação para o cálculo do volume da esfera através do princípio de Cavalieri.

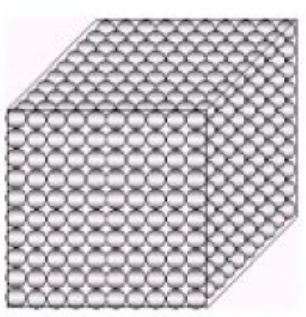

(a)

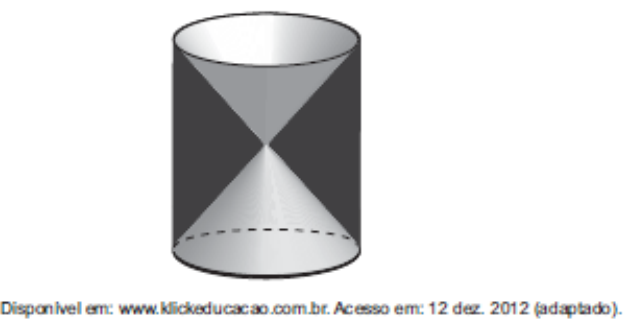

(b)

Figura 1: Questões do ENEM envolvendo a esfera: (a) questão 01 de 1998; (b) questão 170 da Prova Amarela de 2018 7].

Dessa forma, apresentamos neste trabalho duas estratégias que o professor de matemática do Ensino Médio pode utilizar para comprovar a relação para o cálculo do volume da esfera Teorema 2.1]5, 10, e empregamos nessas estratégias o aplicativo de geometria dinâmica GeoGebra 3D 6 6 14 15 18], alinhando assim o planejamento de atividades para a sala de aula ao que estabelece a BNCC sobre o uso de ferramentas computacionais no ensino de geometria.

\section{O volume da esfera}

Teorema 2.1. O volume da esfera $\varepsilon$ de raio $r$ é dado por $\mathcal{V}(\varepsilon)=\frac{4}{3} \pi r^{3}$.

Segundo [8, dentre as estratégias possíveis que o professor de matemática do Ensino Médio pode usar para justificar a relação para o cálculo do volume dos corpos sólidos mais conhecidos, estão o princípio de Cavalier $3^{3}$ e a apresentação clássica de Euclides e Arquimedes

O Princípio 2.1 ou princípio de Cavalieri 8 10, 19,

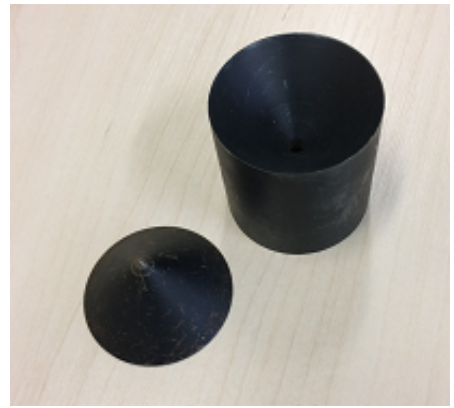

Figura 2: Anticlépsidra e uma das partes da clépsidra em aço carbono [11. reduz o cálculo de volumes ao cálculo de áreas.

Princípio 2.1. Se todo plano paralelo ao plano das bases de dois sólidos, de bases equivalentes e alturas congruentes, determina nos dois sólidos seções equivalentes, então os dois sólidos são equivalentes, ou seja, têm o mesmo volume.

Assim, para usar o princípio de Cavalieri no cálculo do volume de um sólido, precisamos comparar este com um sólido de volume conhecido. No caso da esfera, esse sólido é a anticlépsidra - Figura 2. um cilindro equilátero, de bases congruentes ao círculo máximo da esfera, do qual

\footnotetext{
${ }^{3}$ Bonaventura Francesco Cavalieri (1598-1647): sacerdote e matemático italiano, discípulo de Galileu. É considerado um dos precursores do cálculo integral.

${ }^{4}$ Arquimedes de Siracusa (287 BC-212 BC): matemático, físico, engenheiro, inventor e astrônomo grego. A ele são atribuídas as leis do empuxo e da alavanca.
} 
foram retirados dois cones retos de bases congruentes à base do cilindro e de alturas iguais ao raio da esfera.

Podemos empregar o GeoGebra 3D para mostrar que as seções paralelas aos planos das bases da anticlépsidra e da esfera têm a mesma área - Figura 3 e concluir pelo Princípio 2.1 que o volume da esfera equivale à diferença entre o volume do cilindro equilátero e duas vezes o volume do cone, ou seja:

$$
\begin{aligned}
& \mathcal{V}(\text { esfera })=\mathcal{V}(\text { anticlépsidra })=\mathcal{V}(\text { cilindro equilátero })-2 \mathcal{V}(\text { cone }) \\
& \mathcal{V}(\text { esfera })=\pi r^{2} 2 r-2 \frac{1}{3} \pi r^{2} r=2 \pi r^{3}-\frac{2}{3} \pi r^{3}=\frac{4}{3} \pi r^{3}
\end{aligned}
$$

o que corrobora a tese do Teorema 2.1

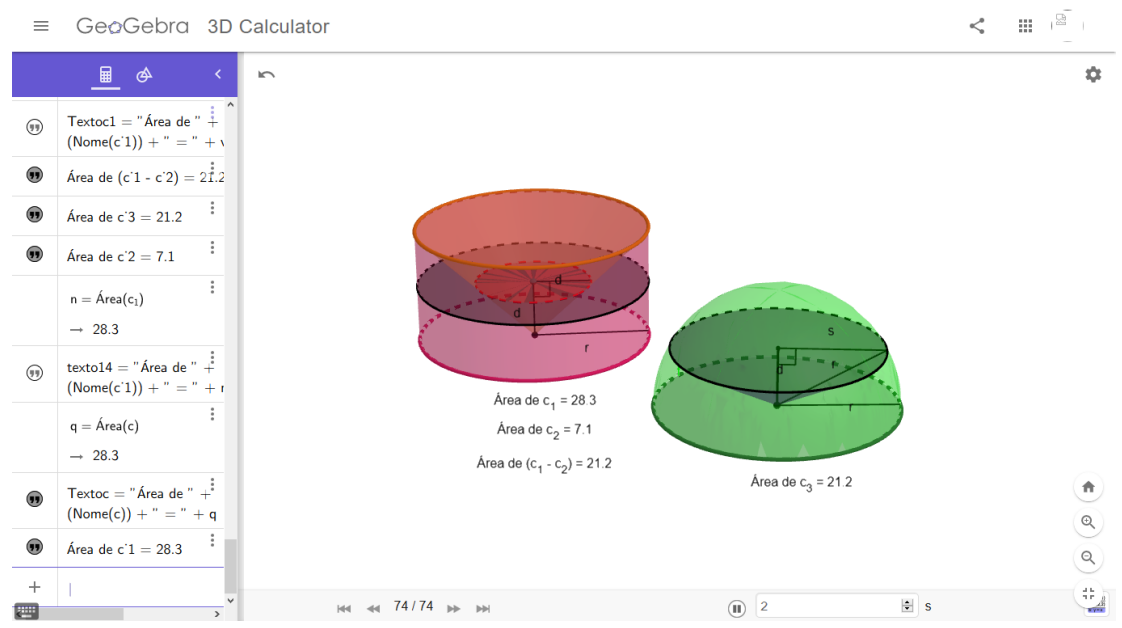

Figura 3: Princípio de Cavalieri: área das seções na anticlépsidra e na esfera no GeoGebra 3D [19].

Contudo, o princípio de Cavalieri, apesar de intuitivo, não pode ser demonstrado de maneira elementar [8]. Assim, o teorema de Arquimedes é outra abordagem que pode ser adotada no Ensino Médio. Na obra O Método [3, Arquimedes descreve uma estratégia mecânica para investigar volumes [1 3, 17, 19, como o volume da esfera descrito por ele no Teorema 2.2. As relações apresentadas neste Teorema são relativas aos sólidos de revolução ilustrados na Figura 4(a), cujos volumes estão na razão $1: 2: 3$.

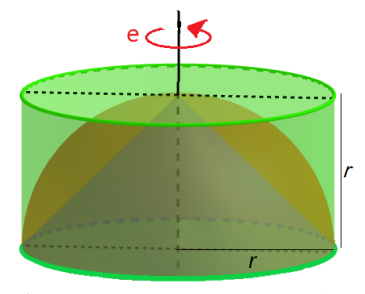

(a)

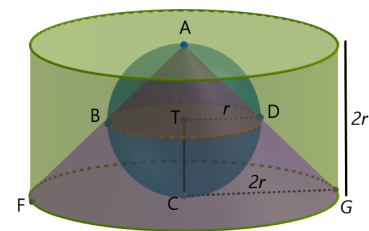

(b)

Figura 4: Volume da esfera: (a) teorema de Arquimedes 19]; (b) comparação dos raios da esfera, do cone e do cilindro na alavanca de Arquimedes 19 . 
Teorema 2.2. O volume de qualquer esfera é igual a quatro vezes o cone que tem sua base igual ao círculo máximo da esfera e sua altura igual ao raio da esfera, enquanto que o volume do cilindro com base igual a um círculo máximo da esfera e altura igual ao diâmetro é uma vez e meia o volume da esfera.

Para provar o volume da esfera segundo Arquimedes [2], precisamos da lei de equilíbrio ou lei da alavanca proposta por Arquimedes na obra Sobre o equilíbrio de figuras planas.

Lei 2.1. Uma alavanca está em equilíbrio se o produto do peso A pela distância a entre o fulcr ${ }^{5}$ e o ponto de supensão de A for igual ao produto do peso $B$ e sua distância $b$ do fulcro, isto é,

$$
\text { A. } a=B . b \quad \text { ou } \quad \frac{A}{B}=\frac{b}{a} .
$$

A Figura 5(a) mostra uma alavanca em equilíbrio, ou seja, uma alavanca onde a relação (1) é respeitada. Empregando essa relação, podemos mostrar que o cilindro de raio e altura $2 r$, a uma distância $d$ do fulcro da alavanca, equilibra o cone de raio e altura $2 r$ e a esfera de raio $r$, ambos a uma distância $2 d$ do fulcro da alavanca 19$]$ - Figura 5 (b).

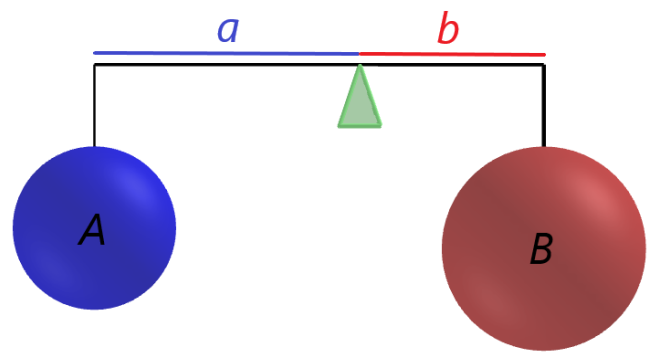

(a)

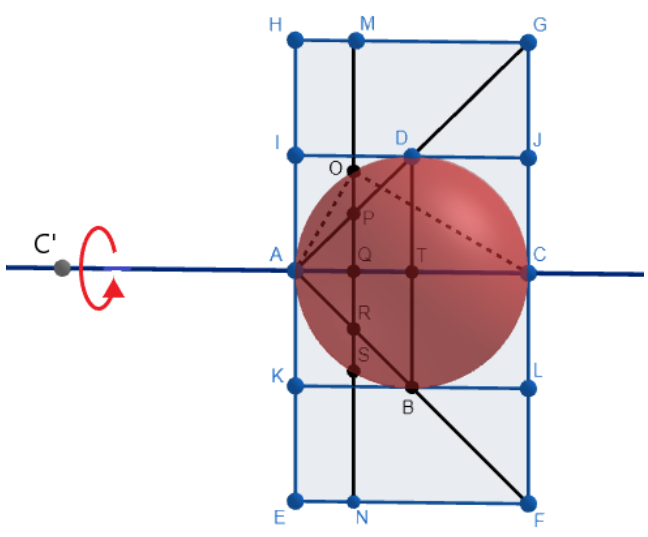

(b)

Figura 5: Lei da alavanca de Arquimedes: (a) princípio de equilíbrio [19; (b) equilíbrio entre cilindro, esfera e cone 19 .

Dessa forma, usando a lei da alavanca e os sólidos ilustrados na Figura 4(b), concluímos que:

$$
\begin{aligned}
\mathcal{V}(\text { cilindro }) d & =[\mathcal{V}(\text { cone })+\mathcal{V}(\text { esfera })] 2 d ; \\
\mathcal{V}(\text { esfera }) & =\frac{1}{2} \mathcal{V}(\text { cilindro })-\mathcal{V}(\text { cone }) \\
\mathcal{V}(\text { esfera }) & =\frac{1}{2} \pi(2 r)^{2} 2 r-\frac{1}{3} \pi(2 r)^{2} 2 r ; \\
\mathcal{V}(\text { esfera }) & =4 \pi r^{3}-\frac{8}{3} \pi r^{3} ; \\
\mathcal{V}(\text { esfera }) & =\frac{4}{3} \pi r^{3}
\end{aligned}
$$

o que confirma a tese do Teorema 2.1.

\footnotetext{
${ }^{5}$ Ponto fixo
} 


\section{Atividades para a sala de aula}

A partir das estratégias para o cálculo do volume da esfera descritas na seção anterior, propomos duas atividades para a sala de aula [19].

Primeiramente, usamos o GeoGebra 3D para organizar uma animação com as várias etapas do cálculo do volume da esfera empregando o princípio de Cavalieri. A Figura 6 mostra a etapa final da animação, que pode ser construída pelos estudantes do Ensino Médio e está disponível em

\section{https://www.geogebra.org/3d/dd4eh7dz}

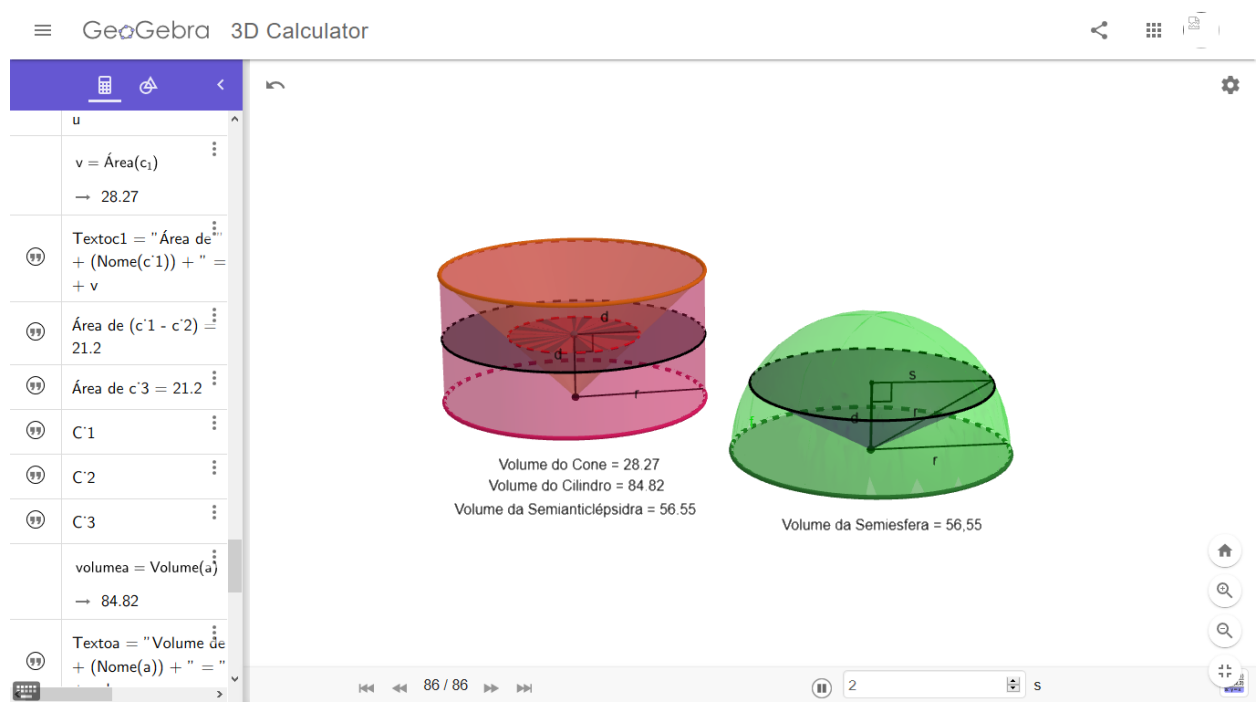

Figura 6: O princípio de Cavalieri: semianticlépsidra e semiesfera equivalentes 19].

Posteriormente, construímos uma balança de Arquimedes - Figura 7- para comparar os volumes de alguns sólidos, particularmente do cilindro, do cone e da esfera. Essa comparação permite deduzir a relação para o cálculo do volume da esfera conhecidas as relações para o cálculo do volume do cilindro e do cone.

Os sólidos ilustrados na Figura 7 foram confeccionados por uma impressora 3D. O experimento ilustrado nessa figura pode ser representado no GeoGebra 3D, como ilustra a Figura 8.

\section{Conclusões}

As estratégias para comprovar a relação para o cálculo do volume da esfera empregadas neste trabalho foram apresentadas aos estudantes do terceiro ano do Ensino Médio do CPM, no qual a segunda autora é professora de matemática. Concluímos que as atividades foram relevantes para construir/consolidar conceitos relativos ao cálculo do volume da esfera.

Esperamos que este trabalho motive os professores de matemática da Educação Básica a comprovar/justificar relações geométricas, ao invés de simplesmente apresentá-las aos estudantes, e também a utilizar aplicativos de geometria dinâmica, como o GeoGebra 3D, nas aulas de geometria. 


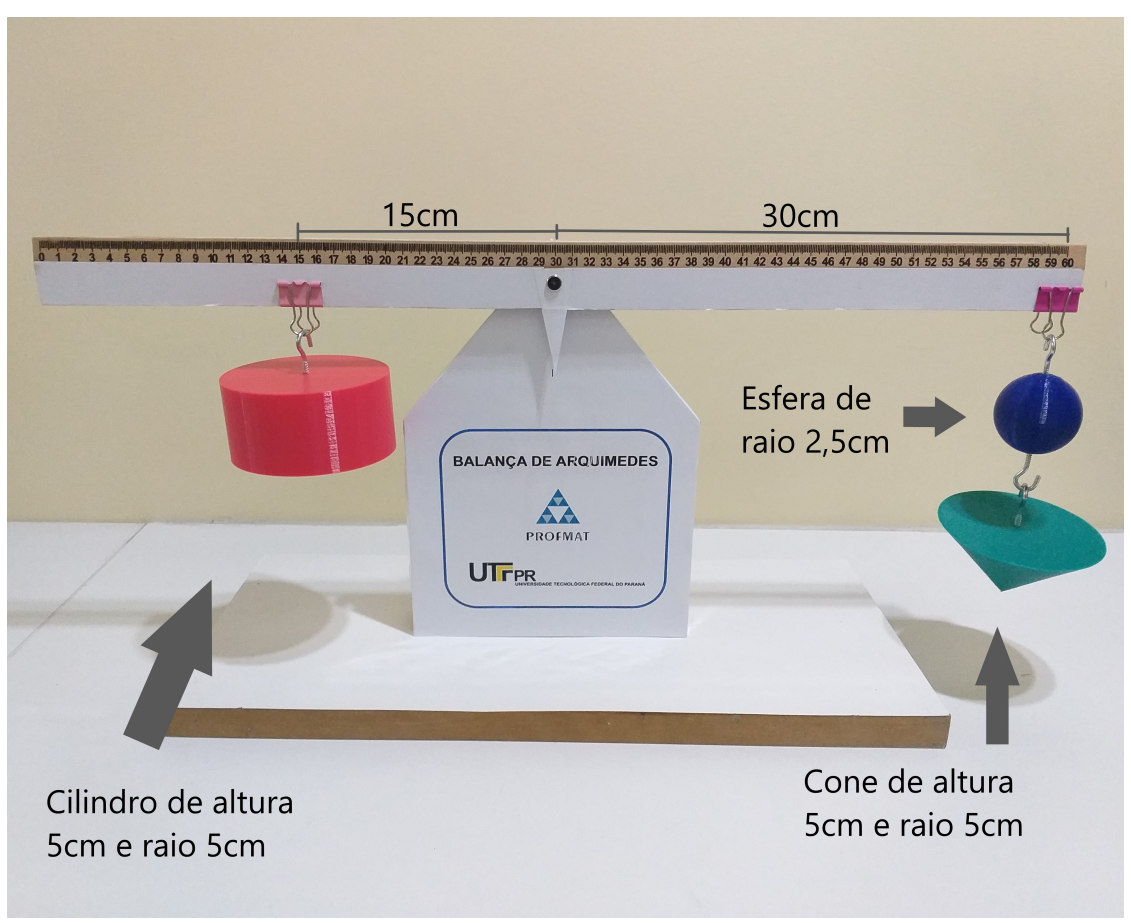

Figura 7: Lei da alavanca de Arquimedes: a esfera e o cone equilibram o cilindro [19.

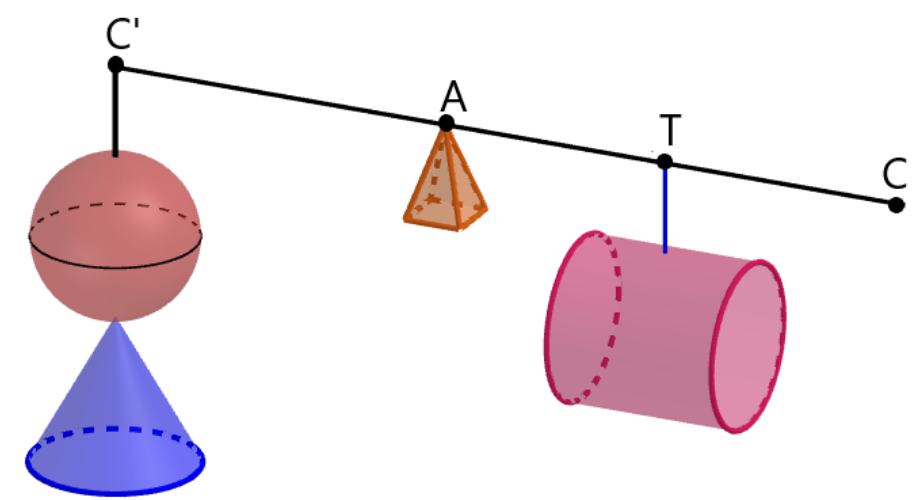

Figura 8: Lei da alavanca de Arquimedes no GeoGebra 3D: a esfera e o cone equilibram o cilindro [19].

\section{Agradecimentos}

O presente trabalho foi realizado com apoio da Coordenação de Aperfeiçoamento de Pessoal de Nível Superior - Brasil (CAPES) - Código de Financiamento 001.

\section{Referências}

[1] Aaobe, A. Episódios da história antiga da matemática, 3. ed. SBM, Rio de Janeiro, 2013. 
[2] Archimedes and Health, T. L. The works of Archimedes. Dover, New York, 1953.

[3] Assis, A. K. T. e Magnaghi, C. P. O método ilustrado de Arquimedes: utilizando a lei da alavanca para calcular áreas, volumes e centros de gravidade. Apeiron, Montreal, 2014.

[4] Brasil. Base Nacional Comum Curricular. MEC/SEB/CNE, Brasília, 2018. Disponível em: http://basenacionalcomum.mec.gov.br/images/BNCC_EI_EF_110518_versaofinal_ site.pdf. Acesso em: 29 dez. 2020.

[5] Dolce, O. e Pompeo, J. N. Fundamentos de matemática elementar: geometria espacial, v. 10, 7. ed. Atual, São Paulo, 2013.

[6] GeoGebra3D. GeoGebra 3D calculator. 2020. Disponível em: https:www.geogebra.org/3d. Acesso em: 29 dez. 2020.

[7] INEP. ENEM - Provas e gabaritos. 2020. Disponível em: http://portal.inep.gov.br/ provas-e-gabaritos. Acesso em: 29 dez. 2020.

[8] Lima, E. L. Medida e forma em geometria, 4. ed. SBM, Rio de Janeiro, 2011.

[9] Lima, E. L. et al. A matemática do Ensino Médio, v. 2, 6. ed. SBM, Rio de Janeiro, 2006.

[10] Neto, A. C. M. Geometria, 1. ed. SBM, Rio de Janeiro, 2013.

[11] Nós, R. L. Acervo de material didático da disciplina Geometria Espacial. UTFPR, Curitiba, 2019.

[12] Nós, R. L. e Fernandes, F. M. Equicomposição de polígonos e o cálculo de áreas, Proceeding Series of the Brazilian Society of Computational and Applied Mathematics, v. 6, n. 2, p. 010272-1 - 010272-7, 2018. DOI: https://doi.org/10.5540/03.2018.006.02.0272.

[13] Nós, R. L. e Fernandes, F. M. Ensinando áreas e volumes por equicomposição, Educação Matemática em Revista, v. 24, n. 63, p. 121-137, 2019. Disponível em: http://sbem.iuri0094. hospedagemdesites.ws/revista/index.php/emr/article/view/1805.

[14] Nós, R. L. e Silva, V. M. R. da. Radicais duplos no cálculo do volume de poliedros convexos, C.Q.D. Revista Eletrônica Paulista de Matemática, v. 16, p. 53-70, 2019. DOI: https://doi. org/10.21167/cqdvol16201923169664rlnvmrs5370.

[15] Nós, R. L. e Silva, V. M. R. da. Compondo/decompondo poliedros convexos com o GeoGebra 3D, Proceeding Series of the Brazilian Society of Computational and Applied Mathematics, v. 7, n. 1, p. 010364-1 - 010364-7, 2020. DOI: https://doi.org/10.5540/03.2020.007.01.0364.

[16] Paraná. Diretrizes Curriculares da Educação Básica - Matemática. Governo do Paraná/SEED/DEB, Curitiba, 2008. Disponível em: http://www.educadores.diaadia.pr. gov.br/modules/conteudo/conteudo.php?conteudo=1. Acesso em: 29 dez. 2020.

[17] Pinto, F. de A. Arquimedes, as alavancas e o volume da esfera, Revista do Professor de Matemática, n. 58, p. 18-20, 2005.

[18] Silva, V. M. R. da e Nós, R. L. Calculando o volume de poliedros convexos. CRV, Curitiba, 2018. DOI: https://doi.org/10.24824/978854442681.4

[19] Tavares, M. C. F. P. Superfícies e sólidos esféricos. Dissertação de Mestrado, UTFPR, Curitiba, 2019. Disponível em: http://repositorio.utfpr.edu.br/jspui/handle/1/4697. 